\title{
GORRESPONDENGE
}

The Editor,

\section{Journal of Glaciology}

SIR,

Thickness change of the Greenland ice sheet:

comments on the letter by B. Federer and H. von Sury

In their recent letter about observations at Jarl Joset Station on the Greenland ice sheet, Federer and von Sury (1976) report that the product of downward velocity $V$ and density $\rho$ is constant for a dozen reference points from $5 \mathrm{~m}$ to $40 \mathrm{~m}$ depth. As they point out, this observation is inconsistent with a model of mine (Nye, 1975) which took Sorge's Law as its starting point.

The difficulty may be expressed as follows. $V \rho$ equals the downward rate of flow of mass across a unit area placed at a fixed height. Thus, if $V \rho$ is constant with depth, it means that, over the period of measurement (1960-68), the downward mass flux measured in a fixed reference frame ( $V$ being absolute velocity) was the same at a dozen depths from $5 \mathrm{~m}$ to $40 \mathrm{~m}$. We can imagine a slab, bounded by horizontal planes at fixed absolute heights, with mass entering through the top surface and leaving through the bottom surface, and we can conclude (conclusion A) that the mass within it, and therefore its mean density, is remaining constant. Now the surface of the ice sheet is lowering, and so getting closer to the slab; that is, the depth of the slab, relative to the snow surface, is decreasing. Consequently, if we assumed Sorge's Law (that the density : depth relation is constant when depth is measured relative to the surface) we should expect the mean density of the slab to decrease, and not stay constant. This is why a model based on Sorge's Law, like mine, is bound to be inconsistent with the observation that $V \rho$ is constant with depth.

To try to explain the observation let us adopt a more refined model, not using Sorge's Law, that takes account of a varying accumulation rate. We know that the accumulation rate has recently $(c$. 1959) decreased significantly. Consider two steady-accumulation (Sorge's Law) density: depth profiles for two different accumulation rates: at a given depth below the surface the density will be higher for the lower accumulation rate (the snow at a given depth has had longer to compact). On this basis we should expect that, with a decreasing accumulation rate, the depth corresponding to a given density will decrease. This is just what the observations show, for this was our conclusion A above: a layer of fixed absolute height, and therefore decreasing depth, retains the same mean density. So the observation that $V \rho$ is constant with depth is not a surprise if one takes account of the decreasing accumulation rate. The observation means that a portion of the density : depth curve, rather than being fixed (Sorge's Law) is translating bodily upwards relative to the surface. The relevant portion of the density curve happens to be fixed in space rather than fixed relative to the surface.

I should particularly like to thank both Dr Federer and Dr von Sury for their most helpful private correspondence on this topic. I was at first inclined to think that the solution to the problem lay in systematic errors in the measurements of density, but it now seems that it might be hard to defend that view.

H. H. Wills Physics Laboratory, J. F. Nye University of Bristol, Bristol BS8 ITL, England I7 August 1976

\section{REFERENGES}

Federer, B., ana Sury, H. [V.] von. 1976. Deducing thickness changes of an ice sheet: comments on the paper by J. F. Nye. Journal of Glaciology, Vol. 17, No. 77, p. 531. [Letter.]

Nye, J. F. 1975. Deducing thickness changes of an ice sheet from radio-echo and other measurements. Journal of Glaciology, Vol. 14, No. 70, p. 49-56. 